\title{
Estudio y Simulación del Beamforming de un Arreglo de Antenas Inteligentes Aplicado en el Sistema de Comunicación Satelital de la Estación Terrena Universidad Surcolombiana Studying and Simulation of a Smart Antennas Array Beamforming Applied in the Satellite Communication System of the Universidad Surcolombiana Ground Station
}

Vladimir Mosquera C. ${ }^{1}$, Harrison René Valencia M. ${ }^{2}$ y Camilo Hernando Botero $\mathrm{S}^{3}$.

\section{Resumen}

Este trabajo presenta el fundamento teórico acerca del proceso de diseño y simulación de arreglos de antenas para comunicaciones satelitales. Se estudian tres distintos arreglos de antenas: Lineal, Plana y el arreglo Cruz de Mills como una alternativa para arreglos de antenas, con su aplicación en estaciones terrenas de monitoreo satelital. Se realiza un estudio del ángulo solido de visibilidad que tiene la estación terrena de la Universidad Surcolombiana, con los requerimientos exigidos por la estación terrena se diseñan los arreglos, verificando su efectividad. Se presenta una herramienta de simulación de arreglos de antenas, esta también permite direccionamiento del haz dependiendo de la posición del satélite. De los arreglos estudiados la Cruz de Mills es el que presenta mejores características para la aplicación satelital.

Palabras Clave: arreglos de antenas; conformación de haz; comunicaciones satelitales; ángulo Solido.

\begin{abstract}
This document provides a theoretical foundation about the design and simulation process of antennas array for satellites communications; three different antennas arrays are shown: Lineal, Planar and the Mills Cross array as alternative for using in antennas arrays, applying this arrays in ground stations for satellites tracking. A study of the solid vision angle of the ground stations of the Universidad Surcolombiana is shown in this document, with the requirements of the ground station the arrays are designed, testing their performance. A simulation tool for arrays antennas is also contributed in this study; this simulation tool also works for feeding of the beam with the satellite position. The array that shows better performance in the satellite application is the Mills Cross.
\end{abstract}

Keywords: antennas arrays; beamforming; satellite communications; solid angle.

1 Ingeniero electronic, MSc.Docente Universidad Surcolombiana Neiva. Avenida Pastrana Carrera 1a. vmosquera@ usco.edu.co. de Investigación en Telemática GITUSCO, Universidad Surcolombiana, Neiva, Colombia.

2 Ingeniero Electrónico, Universidad Surcolombiana Neiva. Avenida Pastrana Carrera 1a. hvalencia@ingenieros.com.

3 Ingeniero Electrónico. Universidad Surcolombiana Neiva. Avenida Pastrana Carrera 1a. camilo.botero@ingenieros.com. 


\section{Introducción}

Las estaciones terrenas se utilizan con el fin de hacer seguimiento y establecer comunicación con satélites [Neri Vela, 2003]. En el marco del proyecto CUBESAT-UD-USCO ${ }^{4}$ se ha construido una estación terrena para el monitoreo de satélites preferiblemente de órbita baja. Dado que la potencia de la señal proveniente de los satélites LEO es muy baja (1 mW) (AMSAT, 2004), se hace necesario inicialmente que el ancho de haz principal del diagrama de radiación de la antena receptora, se reduzca de forma considerable, logrando de esta manera elevada ganancia y directividad. El tipo de antena receptora que usa la estación en estos momentos es una Yagui Uda, con una ganancia de $15 \mathrm{dBi}$ y un ancho del haz de $48^{\circ}$ (Lozano, 2008), características no muy distintas a las diferentes antenas que se utilizan en este tipo de tecnología. En la actualidad se han desarrollado estudios para subsanar problemas existentes en los sistemas de comunicaciones móviles que son similares a los encontrados en las estaciones terrenas satelitales (Covarrubias, 2008).

Una de las propuestas más prometedoras es el empleo de antenas inteligentes en la radio base, particularmente este trabajo se enfoca en la investigación sobre el diseño de agrupamientos de antenas para la conformación de haz (beamforming) de un sistema de antenas inteligentes aplicado a la estación terrena de comunicación satelital USCO (Botero, 2010).

La Estación Terrena fue construida para establecer un canal de comunicación con satélites localizados en la órbita LEO. Este monitoreo de satélites (CUBESAT) se hace con un mecanismo de control que permite posicionar el sistema de antenas terrestres obedeciendo a un modelo basado en los elementos Keplerianos, el cual está asociado en unas herramientas de software que deben simular el comportamiento del satélite y entregar información en tiempo real al sistema de servomotores, con el fin de que éste realice las operaciones físicas de desplazamiento y orientación de las antenas, logrando una alineación con el satélite dando mayor precisión el enlace de comunicación (Lozano, 2008). Sin embargo, este mecanismo presenta una serie de perturbaciones causada por la vibración del rotor, las cuales deterioran el proceso de detección de la señal, no se presenta la información en tiempo real lo que conlleva a tener apuntamientos sin éxito. En consecuencia, el buen desempeño del sistema se ha venido disminuyendo porque las transmisiones satelitales tienen algunas desventajas como los retardos de propagación, la interferencia de radio y microondas y la señal emitida por los pico-satélites es débil (1 mW) (AMSAT, 2004).

Por lo anterior, se hace necesario el uso de un sistema de antenas inteligentes en la estación terrena USCO. El empleo de este sistema se convierte en la tecnología eficaz para solucionar los problemas mencionados. Permitirá no sólo aumentar la capacidad y confiabilidad, sino también mejorar la calidad de la señal, reducir la potencia de transmisión, reducir el nivel de interferencia y dispersión e incluso introducir nuevos servicios aprovechando las particularidades de esta tecnología, la cual se ha utilizado principalmente en telecomunicaciones móviles terrestres (telefonía celular) (Covarrubias, 2008), pero no se conoce hasta el momento registros de aplicaciones a estaciones terrenas satelitales.

Por tal razón, se presenta el modelado y simulación de arreglos de antenas específicos para la conformación de haz (beamforming) de un sistema de antenas inteligentes, aplicado a la estación terrena de comunicación satelital USCO. En este trabajo se consideran algunas agrupaciones uniformes con elementos de antena idénticos en su forma, estructura y radiación, apropiadas para movimientos de satélites en órbitas bajas (LEO), evaluando parámetros del diagrama de radiación como el ancho de haz principal, directividad, nivel de aislamiento, así como el direccionamiento del lóbulo principal hacia una coordenada de interés. Se emplea software especializado en simulación de antenas, y se desarrolla una herramienta software útil para analizar el comportamiento de los diagramas de radiación en las agrupaciones de antenas consideradas sencillas y apropiadas para la aplicación planteada.

Un parámetro de gran importancia en el diseño de arreglos de antenas es la ganancia que se necesita para establecer el canal de comunicación. Estudios anteriores realizados en la estación terrena de la Universidad Surcolombiana (Lozano, 2008), dieron como resultado una serie de valores a ciertos parámetros importantes para el análisis del sistema, entre estos también se tuvieron en cuenta las pérdidas de un sistema de comunicaciones satelital. Dicho estudio da como resultado que la ganancia de la antena de la estación terrena, para el caso particular de la Universidad Surcolombiana, debe tener un valor de $15 \mathrm{dBi}$. Del mismo estudio se determina que el ancho de banda que debe manejar la antena debe ser por lo menos de $10 \mathrm{KHz}$, pero según los datos de la Base de datos de satélites de la comunidad AMSAT consignados en la Tabla II.4 (Apéndice II) de Botero (2010), se debe manejar un mínimo de $100 \mathrm{KHz}$ en ancho de banda para recibir BEACOM de varios satélites, con una frecuencia central $\boldsymbol{f}^{\boldsymbol{o}}=\mathbf{4 3 7 M H z}$.

4 CUBESAT-UD-USCO: la Universidad Surcolombiana (USCO) con la Universidad Distrital Francisco José de Caldas (UD) han trabajo en el área de las comunicaciones satelitales, con el macro-proyecto CUBESAT-UD-USCO telemedicina y telemetría. 
De Lozano (2008) se obtienen anchos de haz a mediana potencia para las antenas que están montadas en la estación terrena, este ancho de haz oscila entre 36 y 50 grados, este valor es muy grande y poco eficiente. Se espera con el arreglo de antenas mejorar este valor. También se obtienen datos de la relación frontal-trasero del diagrama de radiación, este valor oscila entre 20 y $26 \mathrm{~dB}$. La relación de lóbulo principal a secundario tiene que cumplir con una especificación mínima de $10 \mathrm{~dB}$. En las recomendaciones de Lozano (2008) se establece que la polarización de la antena debe ser circular.

\section{Metodología}

\subsection{Análisis de Elevación y Azimut}

Las antenas que forman los sistemas de transmisión y recepción de las estaciones de tierra, tienen un campo de visión limitado, por un lado debido a las restricciones físicas de los dispositivos (ángulo de apertura, potencia) y por otro por la rotación de la Tierra y la órbita que describe el vehículo espacial. De este modo se determina el ángulo sólido de visión de la estación terrena de la Universidad Surcolombiana partiendo de datos estadísticos de pasadas de los satélites de la comunidad AMSAT (AMSAT, 2004) que presentan mejores características; estos son CO-57, RS-22 y el HOPE OSCAR 68.

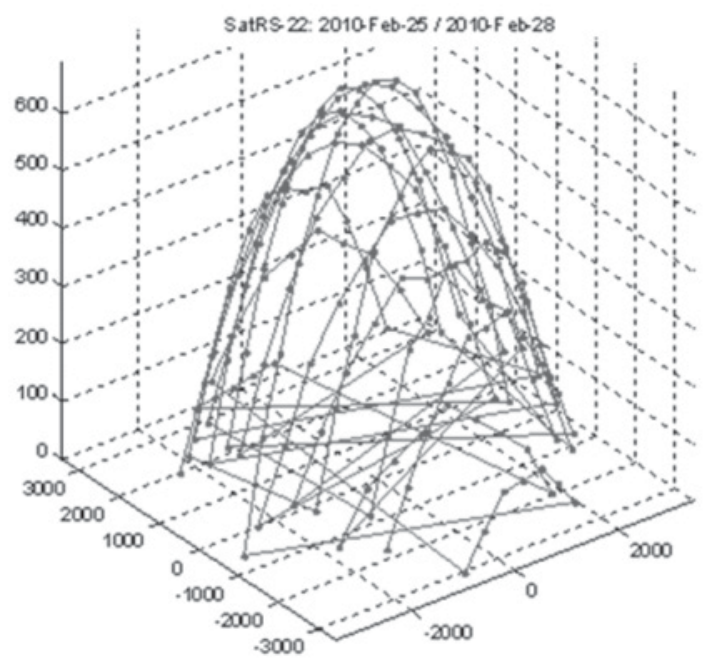

Fig. 1 Gráfico 3D (Az,El,Rg)- SatRS-22: 2010-Feb-25/-Feb-28

En la Figura 1, se presenta un ejemplo de la organización geográfica de los datos. Basados en dichos datos se realiza el análisis estadístico del ángulo de elevación y ángulo de azimut presentando como resultado que el rango óptimo adecuado para la variable elevación está entre 5 a 32 grados. Para la variable azimut el rango óptimo favorable está entre 74 a 285 grados. Con esto queda determinado el ángulo de visión de la estación terrena.

\subsection{Agrupaciones de Antenas}

Una manera de satisfacer los requerimientos exigidos por las comunicaciones satelitales, es formar una estructura de antenas en una configuración eléctrica y geométrica. Esta antena formada por múltiples elementos de antena es conocida como una agrupación (Balanis, 1997). Para simplificar el estudio realizado se consideran los elementos como radiadores isotrópicos ${ }^{5}$.

2.2.1 Agrupación Lineal Uniforme (ALU-Faseado): una agrupación lineal uniforme es aquella en donde los elementos están dispuestos a lo largo de una línea recta con la misma amplitud y fase de excitación, y separados entre sí una distancia d, como se muestra en la Figura 2.

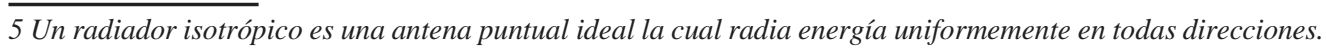




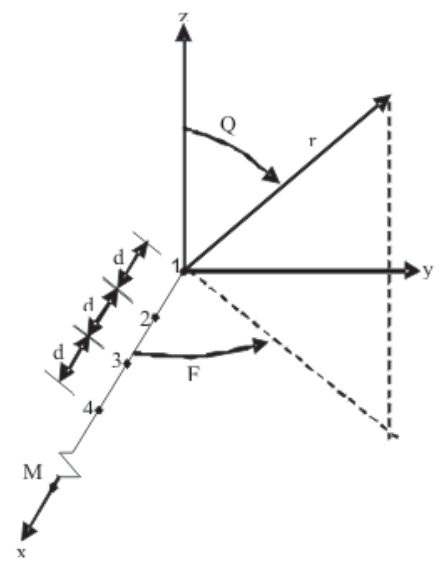

Figura 2 Agrupación lineal uniforme (ALU)

Tomando la amplitud y la fase de cada elemento se define el factor de agrupación (FA) como

$$
\boldsymbol{F A}=\boldsymbol{1}+\boldsymbol{e}^{j(k d \cos (\mathrm{q})+\mathrm{b})}+\boldsymbol{e}^{j 2(k d \cos (\mathrm{q})+\mathrm{b})}+\cdots+\boldsymbol{e}^{j(N-1)(k d \cos (\mathrm{q})+\mathrm{b})}
$$

Haciendo y $=\boldsymbol{k} \boldsymbol{d} \cos \boldsymbol{\theta} \boldsymbol{\boldsymbol { \beta }}$, donde $\theta$ es el ángulo de direccionamiento y $\beta$ es el desfasamiento progresivo de corrientes y el arreglo se ubica en el eje z, se obtiene la siguiente ecuación

$$
\boldsymbol{F A}=\left[\frac{\operatorname{sen}\left(\frac{N}{2} \psi\right)}{\operatorname{sen}\left(\frac{1}{2} \psi\right)}\right]
$$

La Ec. 2 no tiene relación espacio real, pues no considera el movimiento del vector de radiación a lo largo del ángulo $\varphi$, solo lo hace en $\theta$. Para tener una representación real de todo el espacio se recurre al vector de radiación colocando el arreglo en el eje x, realizando el producto punto entre el vector de radiación y el vector del arreglo

$$
\cos \gamma=\hat{a}_{x} \cdot \hat{a}_{r}=\hat{a}_{x} \cdot\left(\hat{a}_{x} \operatorname{sen} \theta \cos \Psi+\hat{a}_{y} \operatorname{sen} \theta \cos \Psi+\hat{a}_{z} \cos \theta\right)=\operatorname{sen} \theta \cos \Psi
$$

quedando el ángulo eléctrico y $=\boldsymbol{k} \boldsymbol{d} \cos [(\cos )]-1(\operatorname{sen} \boldsymbol{\theta} \cos \mathrm{y}))+\beta$, en donde se tiene movimiento en todo el espacio real. Este tipo de arreglo tiene un nivel de lóbulo principal a secundario de $13.2 \mathrm{~dB}$.

Controlando la excitación de las fases entre elementos se puede obtener direccionamientos en cualquier lugar del espacio real. Cuando el arreglo se ubica en el eje z se puede orientar el haz entre $0^{\circ}<<180^{\circ}$, donde $\boldsymbol{\theta}$ es el ángulo de direccionamiento. Para lograr esto el desfase de excitación $\beta$ entre elementos, debe ajustarse de la siguiente manera

$$
\Psi=\boldsymbol{k} \boldsymbol{d} \cos \theta+\beta \mathrm{c}_{\boldsymbol{\theta}_{\theta=\theta \boldsymbol{o}}}=\boldsymbol{k d} \cos \theta_{\boldsymbol{o}}+\beta=O ® \beta=-\boldsymbol{k} \boldsymbol{d} \cos \boldsymbol{\theta}_{\boldsymbol{o}}
$$

Teniendo como ancho de haza mediana potencia

$$
\begin{aligned}
\theta_{h} & =\cos ^{-1}\left(\cos \theta_{o} \frac{-2.782}{N k d}\right)-\cos ^{-1}\left(\cos \theta_{o} \cdot \frac{-2.782}{N k d}\right) \\
D & =\frac{4 \pi U}{P_{r d a}}
\end{aligned}
$$

Donde $U$ es la intensidad de radiación y Prad la potencia de radiación. Desarrollando las expresiones para el factor de agrupación se obtiene que la directividad $D$ está dada por Ec. 7 (ver demostración en apartado 2.2.5.4 de Botero (2010)). 


$$
D_{o}=\frac{1}{\frac{1}{2} \int_{o}^{\pi}\left[\frac{\operatorname{sen}\left(\frac{N}{2} \psi\right)}{N\left(\frac{1}{2} \psi\right)}\right]^{2} \operatorname{sen} \theta d \theta}
$$

(Ec. 7)En Cardama (2002), se demuestra que cuando la separación entre elementos es igual a $\lambda / 2$, se puede considerar que la directividad $D$ es igual al número de elementos $\mathrm{N}$

$$
D=N
$$

Con las ecuaciones encontradas se procede a implementar éstas en algoritmos para posterior simulación del arreglo en MATLAB, con estos algoritmos se pueden encontrar las características eléctricas fundamentales de la antena.

2.2.2 Agrupación plana uniforme: este tipo de arreglos tiene un diagrama de radiación más directivo. A diferencia del diagrama de radiación de una ALU, las agrupaciones planas tienen un solo lóbulo orientado.

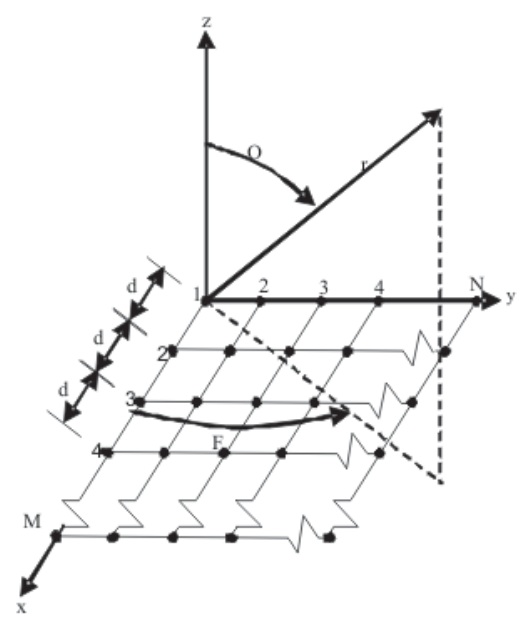

Figura 3. Arreglo plano

La distribución de corrientes en el arreglo es uniforme. Como el arreglo plano es una extensión de un arreglo lineal en x y un arreglo lineal en y, como se observa en la Fig. 3. Se tiene el factor de agrupación (FA) como

$$
F A=\sum_{n=1}^{N} I_{I n}\left[\sum_{m=1}^{N} I_{m l} e^{j(m-l)\left(k d_{x} \operatorname{sen} \theta \cos \varphi+\beta_{x}\right)}\right] e^{j(n-l)\left(k_{y} \operatorname{sen} \theta \operatorname{sen} \varphi+\beta_{y}\right)}
$$

Realizando las mismas consideraciones hechas para la ALU, el factor de agrupación (FA) finalmente se escribe como

$$
F A n(\theta, \varphi)=\left\{\frac{1}{M} \frac{\sin \left(\frac{M}{2} \psi_{x}\right)}{\sin \left(\frac{\psi_{x}}{2}\right)}\right\}\left\{\frac{1}{N} \frac{\sin \left(\frac{N}{2} \psi_{y}\right)}{\sin \left(\frac{\psi_{y}}{2}\right)}\right\}
$$


Donde

$$
\begin{aligned}
& \Psi_{x}=k d_{x} \operatorname{sen} \theta \cos \varphi+\beta_{x} \\
& \Psi_{y}=k d_{y} \operatorname{sen} \theta \cos \varphi+\beta_{y}
\end{aligned}
$$

Del método 18 encontrado en Balanis (1997), se obtiene la representación del ancho de haz a mediana potencia. Basado en este método se obtiene la representación para el ancho de haz de un arreglo plano. El ancho de haz a mediana potencia en el plano de elevación está dado por Ec. 13

$$
\theta_{h}=\sqrt{\frac{1}{\cos ^{2} \theta_{0}\left[\theta_{\mathrm{x} 0}^{-2} \cos ^{2} \phi_{0}+\theta_{\mathrm{y} 0}^{-2} \operatorname{sen}^{2} \phi_{0}\right]}}
$$

donde $\mathrm{q}_{x 0}$ representa el ancho de haz a media potencia de un arreglo lineal tipo transversal de $M$ elementos y $\mathrm{q}_{y 0}$ representa el ancho de haz a mediana potencia del mismo tipo de arreglo pero con $\mathrm{N}$ elementos. El ancho de haz a media potencia en el plano de azimut $\mathrm{Y}_{h}$, se halla de igual manera al de elevación

$$
\Psi \boldsymbol{h}=\sqrt{\frac{1}{\Theta_{x \theta}^{-2} \operatorname{sen}^{2} \phi_{\theta}+\Theta_{y \theta}^{-2} \cos ^{2} \phi_{\theta}}}
$$

Con las expresiones encontradas se puede determinar el ancho del haz a media potencia en los dos planos, azimut y elevación. Es conveniente definir el ángulo solido $\mathrm{W}_{\boldsymbol{A}}$

$$
\Omega_{A}=\frac{\Theta_{y \theta} \Theta_{x o} \operatorname{sen}^{2} \theta_{o}}{\left[\operatorname{sen}^{2} \phi_{o}+\frac{\Theta_{x o}^{2}}{\Theta^{2}{ }_{y \theta}} \cos ^{2} \phi_{o}\right]^{\frac{1}{2}}\left[\operatorname{sen}^{2} \phi_{o}+\frac{\Theta_{y \theta}^{2}}{\Theta_{x o}^{2}} \cos ^{2} \phi_{o}\right]^{\frac{1}{2}}}
$$

La directividad es hallada por medio del ángulo sólido y es equivalente a

$D_{0}=\frac{\pi}{\Omega_{A}\left(\text { rad }^{2}\right)}=\frac{32.400}{\Omega_{A}\left(\text { grados }^{2}\right)}$

(Ec. 16)

Este ángulo sólido se implementa en los algoritmos desarrollados para obtener la dato de directividad, en dichos algoritmos se calculan los anchos de haz para el plano E y para el plano $\mathrm{H}$.

2.2.3 Arreglo plano-Cruz de Mills: un tipo de arreglo plano muy útil particularmente en radioastronomía, es el arreglo conocido como Cruz de Mills. Éste se compone de dos arreglos lineales colocados perpendicularmente y ubicados simétricamente respecto del punto central, como se muestra en la Figura 4.

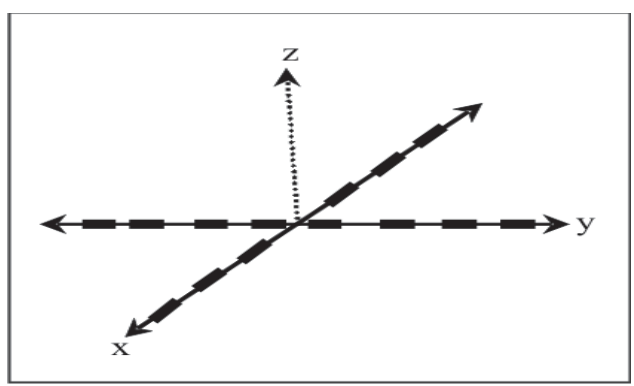

Figura 4 Arreglo plano- Cruz de Mills 
Facultad de Ingeniería Universidad Surcolombiana

El campo total producidos por el arreglo, es equivalente a la suma de los campos individuales de cada arreglo, es decir,

$E_{T}=E_{x}+E_{y}$

Se configura el factor de arreglo de la Cruz de Mills como

$$
\boldsymbol{F A}=\frac{\operatorname{sen}\left(\frac{N}{2} \psi_{1}\right)}{\operatorname{sen}\left(\frac{1}{2} \psi_{1}\right)}+\frac{\operatorname{sen}\left(\frac{N}{2} \psi_{2}\right)}{\operatorname{sen}\left(\frac{1}{2} \psi_{2}\right)}
$$

Donde

$\psi \boldsymbol{x}=\boldsymbol{k d} \operatorname{sen} \theta \cos \psi+\beta \boldsymbol{I}_{\theta=\theta 0^{\prime} \psi=\psi_{0}} \mathrm{y} \psi \boldsymbol{x}=\boldsymbol{k d} \operatorname{sen} \theta \operatorname{sen} \psi+\beta \boldsymbol{I}_{\theta=\theta 0^{\prime} \psi=\psi \theta}$

El ancho de haz y la directividad se obtienen de manera similar al arreglo plano uniforme, teniendo en cuenta que la Cruz de Mills tenga un número de elementos considerables (es recomendable que sea mayor a 20 elementos en cada eje).

Su implementación en un algoritmo en MATLAB resulta ser sencilla, pues se emplea el código utilizado para la ALU teniendo en cuenta que son dos arreglos lineales ubicados perpendicularmente. Se utilizan los algoritmos del arreglo plano para obtener el dato de ancho de haz a mediana potencia y directividad.

\section{Resultados}

Teniendo presente los parámetros ampliamente mencionados en el Capítulo 2 [Botero, 2010]. La simulación realizada en MATLAB se basa en obtener el diagrama de radiación, es decir, la conformación de haz (Beamforming) para los tipos de agrupación de antenas estudiados, de acuerdo a parámetros establecidos al inicio de la simulación. Los algoritmos desarrollados se encuentran en el Apéndice I de Botero (2010).

Por generalización de los estudios se utiliza la antena que se considera como base de las agrupaciones y en el momento de implementar es económica, esto quiere decir que si se cumple las características con esta antena, al cambiarla por otra muy seguramente van a mejorar las características. La antena que se escoge para este fin es la antena dipolo cruzado $\lambda / 2$.

\subsection{Simulación ALU (Arreglo Lineal Uniforme- Faseado)}

En las Figuras. 5 y 6 , se observa la simulación del diagrama de radiación para una ALU de 10 dipolos $\lambda / 2$ cruzados, posicionando su haz principal en una dirección específica del espacio. La ALU simulada está situada a lo largo del eje $\mathrm{x}$.

Direccionamiento $\theta=45^{\circ}$ y $\phi=45^{\circ}$, esto quiere decir $\beta=90^{\circ}$.

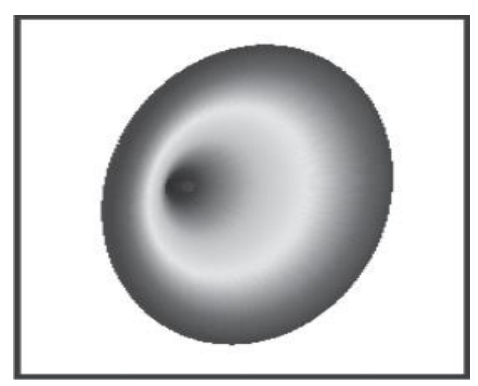

Figura 5 Diagrama 3D de un arreglo ALU 

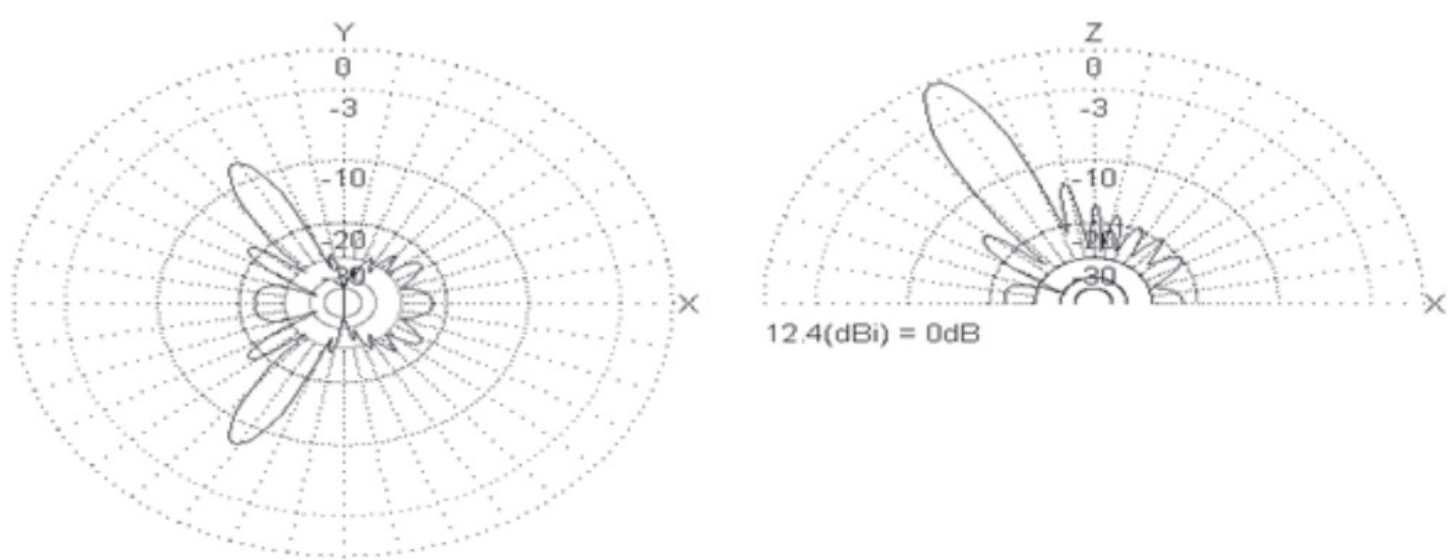

Figura 6 Corte plano E - Corte plano H

Como se observa en las Figuras 5 y 6, en arreglos de tipo ALU, pese a que se pueden direccionar a cualquier lugar en el espacio, tienen un diagrama de radiación que se asemeja a la figura de un cono, lo cual no es conveniente para el tipo de comunicación que se está trabajando, pues se tendría interferencias de las distintas señales que coincidan con el lóbulo cónico de radiación de la ALU.

\subsection{Simulación Arreglo plano uniforme}

2.5 Direccionamiento $\theta=60^{\circ}$ y $\phi=30^{\circ}$, esto quiere decir $b_{x}=78^{\circ}, b_{y}=135^{\circ}$.

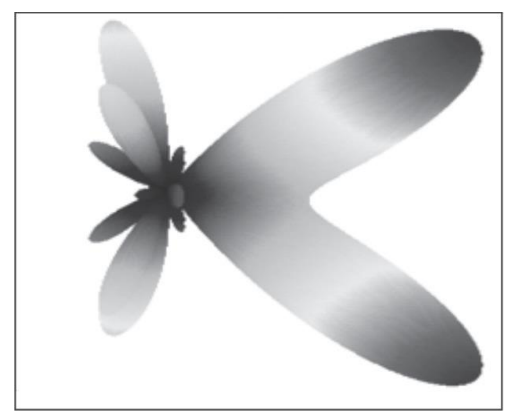

Figura 7 Diagrama 3D Arreglo plano 5X5 dipolos
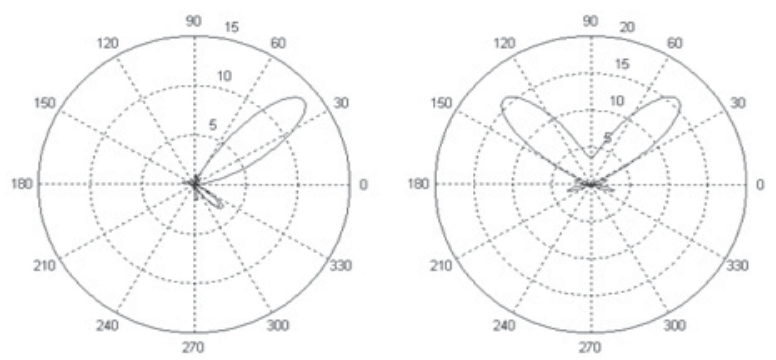

Figura 8 Corte plano E - Corte plano H

Buscando reducir las señales de interferencia en el canal de comunicación, se presenta la simulación de un arreglo plano en las Fig. 7 y Fig. 8, el cual tiene un solo lóbulo principal, evitando la radiación en forma de cono presentada por la ALU. A continuación se muestra la simulación de un arreglo plano de 5x5 con alimentación uniforme en cada uno de sus elementos. Este tipo de arreglos son costosos en su implementación, pues se requieren desfasadores en cada uno de los puntos de su matriz. 
En arreglos planos el espaciamiento entre los elementos cumple un papel fundamental, ya que distancias mayores a media longitud de onda, aparecen en el diagrama de radiación lóbulos secundarios que tiene potencia similar al lóbulo principal, lo cual lleva a un desperdicio de la energía y a problemas en la recepción de señales no deseadas. Se concluye entonces que para arreglos planos el mejor espaciamiento es el de media longitud de onda.

\subsection{Simulación Arreglo plano-Cruz de Mills}

En las Fig. 9 y Fig. 10, se muestra la simulación del arreglo Cruz de Mills para N=10 elementos en cada eje, posicionados sobre el plano $\mathrm{x}-\mathrm{y}$, con distribución de corriente uniforme para cada elemento, elementos espaciados a una distancia de $\lambda / 2$ y con desfase progresivo en cada eje.

Direccionamiento $\theta=45^{\circ}$ y $\phi f=45^{\circ}$, esto quiere decir $b_{x}=90^{\circ}, b_{y}=90^{\circ}$.

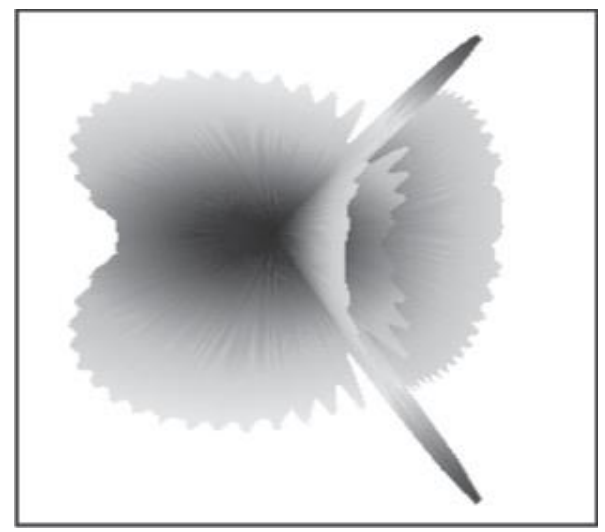

Figura 9 Diagrama 3D Cruz de Mills, $b_{1}=90^{\circ}, b_{2}=90^{\circ}, \mathrm{N}=10$

Cumpliendo el mismo principio que en los arreglos planos, se ve que en el arreglo Cruz de Mills, el espaciamiento óptimo es el de media longitud de onda. Se observa en las Figura 9 y Figura 10, como el diagrama de un arreglo Cruz de Mills tiene lóbulos secundarios que podrían incidir en la calidad de la señal de recepción, pero se concluye que con un número de elementos superior a 10 en cada eje, estos lóbulos secundarios pierden importancia respecto al lóbulo principal. Se presenta este arreglo como la mejor geometría a implementar en estaciones terrenas, esto debido a su buen comportamiento eléctrico y a que no es tan costoso su montaje como lo son los de las otras geometrías.
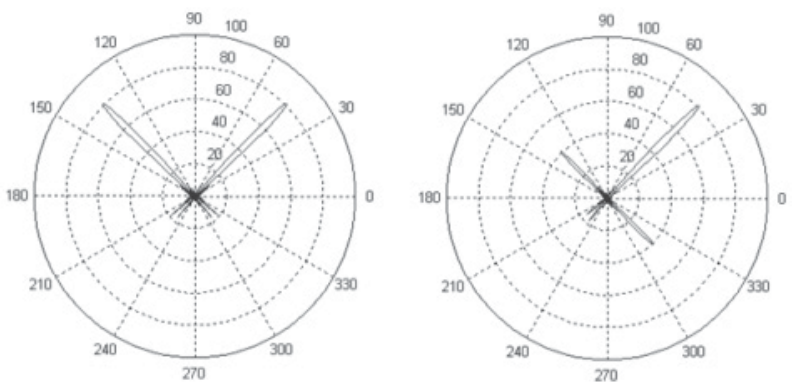

Figura 10 Corte plano $\mathrm{E}$ - Corte plano $\mathrm{H}, \mathrm{b}_{1}=90^{\circ}, \mathrm{b}_{2}=90^{\circ}, \mathrm{N}=10$

Para los arreglos examinados se desarrollaron algoritmos de diseño y se trasladaron a scripts en Matlab, los cuales fueron estructurados como rutinas para trabajarlos desde un programa principal de interfaz gráfica GUIDE de MATLAB. Las ventanas principales del software desarrollado se muestran en la Figura 11. 

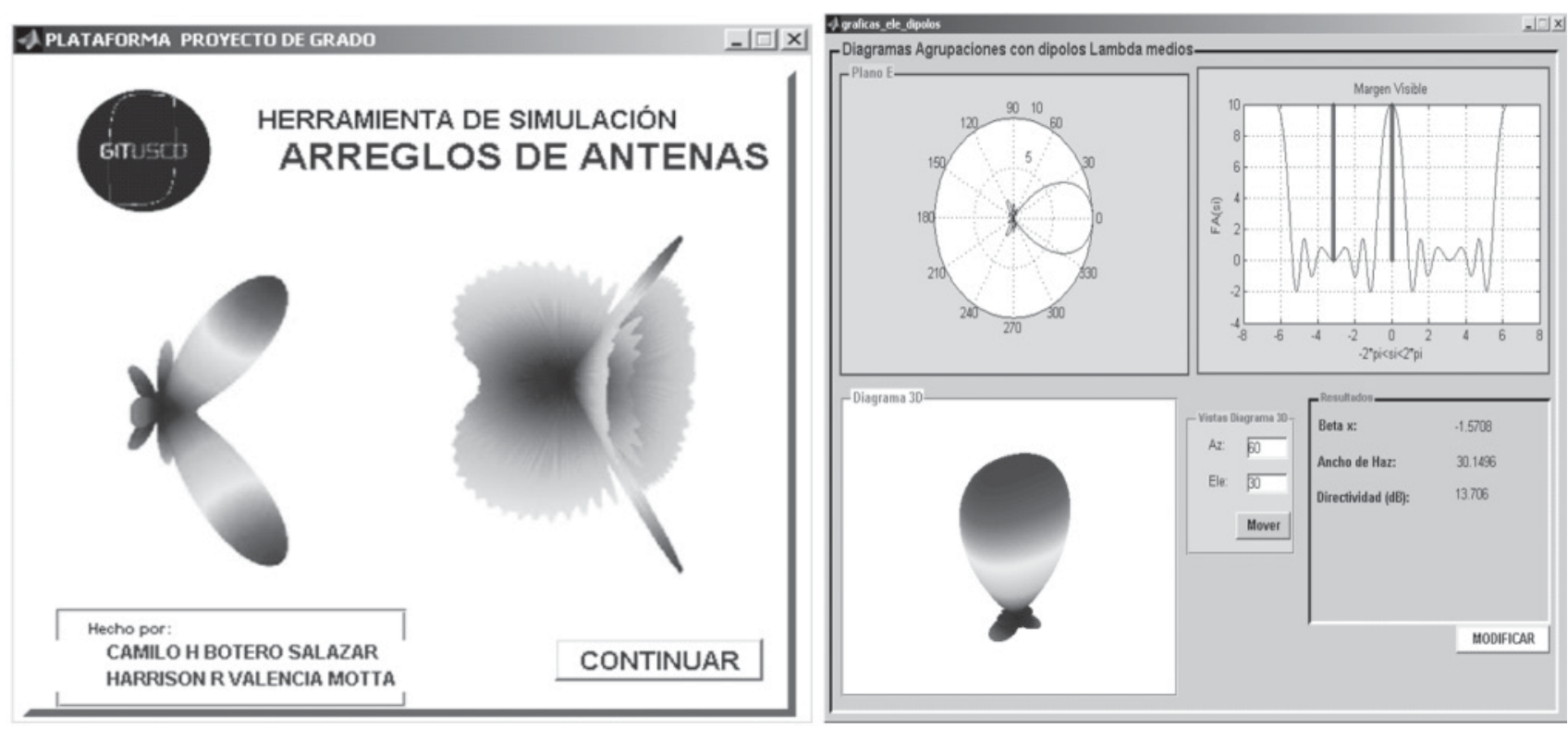

Figura 11 Ventana de presentación - Ventana diagramas de radiación arreglos con dipolos

El software permite comprender y analizar fácilmente el comportamiento de los diagramas de radiación en los tipos de agrupaciones de antenas estudiadas, en base a fórmulas y modelos matemáticos previamente demostrados.

\section{Conclusiones}

Se confirmó que si el espacio entre elementos aumenta lo suficiente para que los máximos periódicos entren en margen visible aparecen lóbulos de difracción. De forma contraria, al modificar la fase progresiva $\beta$, se apreció que el rango del margen visible no cambia, pero se desplaza dependiendo del valor de la fase. Con lo cual, al aumentar la fase progresiva el máximo se desplaza de la dirección transversal al eje del arreglo y el ancho de haz aumenta de forma significativa.

A medida que se incrementa el espaciamiento entre elementos, manteniendo un número fijo de elementos, se obtiene un lóbulo principal más directivo pero con un número más creciente de lóbulos laterales y nulos. De igual forma se demostró que a medida que se obtiene un lóbulo principal más directivo se incrementa el número de lóbulos laterales y de nulos del patrón direccional. En consecuencia, son fundamentales los dos parámetros: espaciamiento entre elementos y número de elementos, para definir las prestaciones de un arreglo de antenas en razón a la aplicación planteada.

Como aportes originales se presenta un estudio estadístico de la ubicación de satélites de la comunidad AMSAT, con características apropiadas con respecto a la estación terrena USCO. Esto con el fin de establecer demostrativamente el cono de visión efectivo para el apuntamiento electrónico generado por la conformación de haz en $(\theta, \varphi)$.

Se contribuye con los análisis hechos para cada una de las tres configuraciones lineal, arreglo plano rectangular y arreglo plano cruz de Mills y el efecto en su patrón de radiación al cambiar parámetros como número de elementos y separación entre ellos.

En la literatura siempre se encuentra el modelo de un arreglo lineal de antenas ubicado en el eje z. Se determinó el factor de arreglo para un arreglo lineal teniendo la consideración de que este está ubicado en el eje x. Se observa como el diagrama de radiación varía según los requerimientos, en todas las direcciones del espacio. 
Facultad de Ingeniería Universidad Surcolombiana

\section{Referencias Bibliográficas}

1. AMSAT., (2004). Operational OSCAR Satellite Status Summary. Obtenida el 2 de febrero de 2010. The Radio Amateur Satellite Corporation: http://www.amsat.org/amsatnew/satellites/status.php

2. Balanis, C. A., (1997). Antenna Theory Analysis and Design. 2 ed. New York: Wiley interscience. $1045 \mathrm{p}$.

3. Botero, C., Valencia, H., (2010). Estudio y Simulación del Beamforming de un Arreglo de Antenas Inteligentes Aplicado en el Sistema de Comunicación Satelital de la Estación Terrena Universidad Surcolombiana. Neiva, 13p. Trabajo de, grado (Ingeniero Electrónico). Universidad Surcolombiana. Facultad de Ingeniería Electrónica. Área de Comunicaciones.

4. Cardama, A., (2002). Antenas. 2 ed. Barcelona: Edicions UPC. 468 p.

5. Covarrubias, D., (2008). Notas del Curso de Antenas Inteligentes para Comunicaciones Celulares. CICESE.

6. Lozano, D., Velásquez, O., (2008). Implementación y Puesta en Funcionamiento del Sistema de Comunicaciones de la Estación Terrena CUBESAT USCO. Neiva, 112 p. Trabajo de grado (Ingeniero Electrónico). Universidad Surcolombiana. Facultad de Ingeniería Electrónica. Área de Comunicaciones.

7. Neri vela, R., (2003). Comunicaciones por Satélite. México: International Thomson Editores. $544 \mathrm{p}$.

8. Ospina, M., (2006). Diseño de un Sistema de Antena Inteligente para una Celda PCS. Bogotá D.C., 70 p. Trabajo de grado (Magister en Ingeniería - Telecomunicaciones). Universidad Nacional. Facultad de Ingeniería. Maestría en Telecomunicaciones. Departamento de Ingeniería de Sistemas e Industrial.

9. Ramos, J., Martínez, M., Ruiz, M., (2002). Arreglos Planos: la Cruz de Mills. 8a., Conferencia de Ingeniería

Electrónica: México D.F., 6 p. Universidad de Guadalajara. Departamento de Electrónica, CUCEI. 